\section{Barreiras, para a notificação pelo pediatra, de maus-tratos infantis}

\author{
Barriers for reporter of child abuse \\ by pediatricians
}

\author{
Joelza M. Pires 1 \\ Marcelo Zubaran Goldani 2 \\ Elisabeth M. Vieira 3 \\ Tiago R. Nava 4 \\ Letícia Feldens 5 \\ Kelly Castilhos 6 \\ Vinícius Simas 7 \\ Núbia S. Franzon 8
}

1,2,4-8 Departamento de Pediatria e Puericultura.

Faculdade de Medicina. Universidade Federal do Rio Grande do Sul. Rua Ramiro Barcelos, 2400. Porto Alegre, RS, Brasil. CEP: 90.035-003 E-mail: mgoldani@hcpa.ufrsg.br

3 Departamento de Medicina Social. Faculdade de Medicina de Ribeirão Preto da Universidade de São Paulo, SP, Brasil

\section{Resumo}

Objetivos: determinar os fatores que interferem na notificação de maus-tratos infantis, pelos pediatras, aos serviços de proteção à criança.

Métodos: estudo observacional transversal. Uma amostra aleatória de pediatras de Porto Alegre foi selecionada entre 990 inscritos na sociedade de pediatria local. Variáveis sócio-demográficas, formação profissional, conhecimento diante de casos de maustratos infantis foram obtidos através de questionário anônimo. Análises descritiva e multivariada foram utilizadas para determinar os fatores associados a não notificação.

Resultados: foram incluidos 97 pediatras dos quais 92 concordaram em participar do estudo. Oitenta identificaram casos de maus-tratos, e destes 63 notificaram ao menos um caso. A maioria revelou medo de envolver-se legalmente, apresentou nivel suficiente de conhecimento e baixo grau de confiança nos órgãos de proteção à criança. Conhecimento insuficiente $(O R=3,94)$, trabalhar exclusivamente no setor privado $(O R=6,33)$ foram fatores associados $a$ não notificação. Após ajustes, o conhecimento insuficiente foi significativamente associado com o resultado $O R=5,06(I C 95 \%=1,45-17,59)$.

Conclusões: verificou-se uma alta taxa de identificação e notificação, pelo pediatra, de maus-tratos infantis. Programas de educação continuada, melhoria dos serviços de proteção, suporte técnico profissional para o setor privado podem aumentar a taxa de identificação e notificação de maus-tratos.

Palavras-chave Maus-tratos infantis, Violência doméstica, Notificação de abuso 


\section{Introdução}

A notificação mandatória de maus-tratos na infância foi instituída no Brasil no início da década de 90, através do Estatuto da Criança e do Adolescente.1,2 Segundo essa lei, toda a suspeita de maus-tratos contra crianças e adolescentes deverá ser obrigatoriamente notificada aos órgãos de proteção. O pediatra encontra-se freqüentemente diante do dilema da notificação, sofrendo pressões legais, profissionais e emocionais que podem influenciar sua decisão.

Estudos avaliando atitudes de médicos em países que possuem legislação semelhante apontam uma taxa de notificação inferior aos casos identificados (teoricamente essa relação deveria ser 1:1).3,4 Dentre os vários fatores que interferem na não notificação de maus-tratos infantis, pelo médico, citamse: o tipo e o grau de gravidade dos maus-tratos, o conhecimento insuficiente sobre os procedimentos de notificação, a deficiência de estruturas de atendimento, influências culturais, experiências prévias, desconfiança nos serviços de proteção à criança, medo de serem processados e envolvimento emocional com a família da criança vítima. ${ }^{5-6}$

Nesses estudos notou-se que o pediatra teria uma propensão a subavaliar a gravidade dos maus-tratos quando comparado com outros trabalhadores da saúde, notificando-os com menor freqüência. ${ }^{7-9} \mathrm{O}$ tipo, a magnitude da gravidade, a idade da criança, a explicação dos maus-tratos pelos pais, a percepção do pediatra da gravidade dos casos foram outros fatores envolvidos na atitude de notificar. $5,10,11$

No Brasil não há estudos quantitativos sobre fatores relacionados à identificação e notificação pelos pediatras de maus-tratos infantis. Em um estudo qualitativo o despreparo técnico e a desconfiança nos órgãos de proteção foram apontados como as maiores dificuldades para a notificação. ${ }^{12}$

Considerando a importância do tema avaliou-se a relação entre identificação e notificação de maustratos contra crianças e adolescentes, como também fatores que interferem no diagnóstico e posterior notificação pelo pediatra.

\section{Métodos}

Um estudo observacional transversal foi feito a partir de uma amostra aleatória de pediatras de Porto Alegre, Rio Grande do Sul, Brasil. O cálculo do tamanho da amostra foi realizado considerando a população de 990 pediatras registrados na Sociedade de Pediatria do Rio Grande do Sul. Um tamanho amostral com 92 pediatras permitiria detectar diferenças de $20 \%$ na taxa de notificação entre grupos de pediatras, com grau de conhecimento suficiente e insuficiente, com intervalo de confiança de $95 \%$ ou $\mathrm{p}<0,05$, e um poder de teste de $80 \%$.

Um questionário padronizado foi desenvolvido para estudar a posição do pediatra diante de casos de maus-tratos infantis, avaliando a influência das características demográficas do profissional, do conhecimento e da história pessoal sobre a atitude de notificar um caso de maus-tratos. A consistência interna do questionário foi avaliada por meio de estudo piloto, apresentando um alfa de Crombach $=$ $0,72.13$

As variáveis sócio-demográficas avaliadas foram: idade (anos completos), sexo, ter filhos (sim ou não), experiência profissional ( $<11$ anos, 11 anos a 20 anos, $>20$ anos), tipo de faculdade cursada (pública ou privada), grau de formação (especialização, residência, mestrado, doutorado, nenhuma), história de ter ele mesmo sofrido algum tipo de maus-tratos quando criança (sim ou não).

$\mathrm{Na}$ avaliação do grau de conhecimento do pediatra sobre maus-tratos utilizaram-se 21 questões considerando aspectos relacionados ao diagnóstico, tratamento e prevenção. Foram utilizadas vinhetas baseadas em casos verídicos acompanhados num serviço de proteção à criança, selecionando histórias de maus-tratos sexual, físico, emocional e negligência. As questões com a primeira e a segunda maiores taxas de erro receberam valores três e dois respectivamente, totalizando 24 acertos possíveis. $\mathrm{O}$ grau de conhecimento foi classificado da seguinte forma: insuficiente (0-17 acertos), suficiente (18-24 acertos). Foram avaliados o grau de confiança nos órgãos de proteção à criança (confia e não confia) e o medo de envolvimento legal (sim e não).

Os pediatras foram contatados pelo telefone por estudantes de medicina previamente treinados. Caso concordassem em responder o questionário, este lhes era entregue pessoalmente, sendo posteriormente recolhido sem identificação sendo colocado em uma urna.

Os dados foram analisados por meio de estatística descritiva com intervalo de confiança de $95 \%$ (IC95\%). Foi realizada uma análise multivariada de regressão logística para determinar as barreiras para a não notificação dos casos de maus-tratos. Também foi incluída no modelo logístico a variável associada significativamente à notificação na análise bivariada. Foi considerado não-notificação o fato de o pediatra informar haver identificado, ou suspeitado, de pelo menos um caso de maus-tratos nos últimos cinco anos, porém não o notificar aos órgãos de proteção. 
A avaliação dos aspectos éticos foi realizada pela Comissão de Ética do Grupo de Pesquisa e PósGraduação do Hospital de Clínicas de Porto Alegre.

\section{Resultados}

Foram selecionados 129 pediatras, porém 32 não preencheram os critérios de inclusão por mudança de cidade, especialidade ou morte. Dos 97 pediatras elegíveis, cinco se negaram a participar da pesquisa, caracterizando uma perda de $5,15 \%$.

Dos restantes 92 pediatras, $80(86,96 \%)$ informaram ter identificado pelo menos um caso de maus-tratos e, entre esses $63(78,75 \%)$ o notificaram aos órgãos de proteção à infância. Maus-tratos físicos foram identificados por $63(78,75 \%)$; sexuais por $57(71,25 \%)$, emocionais por $33(41,25 \%)$ e negligência por $58(72,5 \%)$ dos pediatras. Após análises bivariadas o grau de conhecimento, o tempo e o local da atividade profissional não mostraram influencia na possibilidade de identificar um caso de maus-tratos infantis $(p>0,05)$.

As características sócio-demográficas apontam para um predomínio significativo do sexo feminino, em que a mediana da idade foi de 42 anos (26 a 76 anos). A maioria teve acesso à formação em faculdades públicas, residência médica e atua predominantemente no setor público. A mediana do tempo de formação na graduação foi de 17,5 anos (3 a 47 anos). (Tabela 1)

A maior parte dos pediatras apresentou um grau de conhecimento sobre maus-tratos infantis suficiente, apesar de a maioria deles não ter realizado treinamento específico nessa área; mais da metade declarou não confiar nos órgãos de proteção às crianças e, 94,6\%, relatou temer sansões legais por notificar um caso de maus-tratos infantis. (Tabela 2)

Entre os fatores associados à não notificação destaca-se o grau de conhecimento insuficiente e o fato de trabalhar no setor exclusivamente privado. Após ajuste, apenas a falta de conhecimento relacionou-se independentemente à não notificação OR $=5,06($ IC95\% $=1,45-17,59)$. (Tabela 3$)$

Em relação a ter sofrido algum tipo de violência na infância, $11(11,06 \%)$ dos pediatras relataram terem sido vítimas de maus-tratos na infância. Desses, apenas $50 \%$ notificou casos de maus-tratos identificados em seus pacientes.
Tabela 1

Freqüência das variáveis sócio-demográficas e pessoais.

\begin{tabular}{|c|c|c|c|}
\hline Variável & $N=92$ & $\%$ & IC95\% \\
\hline \multicolumn{4}{|l|}{ Sexo } \\
\hline Masculino & 36 & 39,13 & $29,12-49,86$ \\
\hline Feminino & 56 & 60,87 & $50,14-70,88$ \\
\hline \multicolumn{4}{|l|}{ Setor de atividade } \\
\hline Público e privado/público & 77 & 83,70 & $74,53-90,57$ \\
\hline Privado & 15 & 16,30 & $9,42-25,46$ \\
\hline \multicolumn{4}{|l|}{ Filhos } \\
\hline Não & 34 & 36,96 & $27,12-47,65$ \\
\hline Sim & 58 & 63,04 & $52,34-72,88$ \\
\hline \multicolumn{4}{|l|}{ Pós-graduação } \\
\hline Especialização & 8 & 8,70 & $3,83-16,42$ \\
\hline Residência & 67 & 72,83 & $62,55-81,58$ \\
\hline Mestrado/doutorado & 15 & 16,30 & $9,42-25,45$ \\
\hline Nenhuma & 2 & 2,17 & $0,26-7,63$ \\
\hline \multicolumn{4}{|l|}{ Universidade de graduação } \\
\hline Pública & 74 & 80,43 & $70,85-87,97$ \\
\hline Particular & 17 & 18,48 & $11,15-27,92$ \\
\hline Não informou & 1 & 1,09 & $0,03-5,91$ \\
\hline \multicolumn{4}{|l|}{ Tempo de formado } \\
\hline$<11$ anos & 26 & 28,26 & $19,36-38,61$ \\
\hline $11-20$ anos & 29 & 31,52 & $22,23-42,04$ \\
\hline$>20$ anos & 34 & 36,96 & $27,12-47,65$ \\
\hline Não informou & 3 & 3,26 & $0,68-9,23$ \\
\hline \multicolumn{4}{|l|}{ Sofreu maus-tratos } \\
\hline Sim & 11 & 11,09 & $6,12-20,39$ \\
\hline Não & 80 & 86,96 & $78,32-93,07$ \\
\hline Não responderam & 1 & 1,09 & $0,03-5,91$ \\
\hline
\end{tabular}

Tabela 2

Grau de conhecimento e treinamento sobre maus-tratos infantis, confiança nos órgãos de proteção à criança e temor de envolvimento legal com o caso.

\begin{tabular}{lccc}
\hline Variável & $\mathbf{N}=\mathbf{9 2}$ & $\%$ & IC95\% \\
\hline $\begin{array}{l}\text { Conhecimento } \\
\quad \text { Suficiente } \\
\quad \text { Insuficiente }\end{array}$ & 54 & & \\
$\quad$ Treinamento & 38 & 58,70 & $47,94-68,86$ \\
$\quad$ Sim & & 41,30 & $31,13-52,05$ \\
$\quad$ Não & 44 & 47,83 & $37,30-58,50$ \\
Confiança & 48 & 52,17 & $41,50-62,70$ \\
$\quad$ Sim & & & \\
$\quad$ Não & 38 & 41,30 & $31,13-52,05$ \\
$\quad$ Não sei & 49 & 53,26 & $42,56-63,74$ \\
Medo de envolvimento legal & 5 & 5,43 & $1,79-12,23$ \\
$\quad$ Sim & 87 & & \\
$\quad$ Não & 5 & 94,57 & $87,77-98,21$ \\
& & 5,43 & $1,79-12,23$ \\
\hline
\end{tabular}


Razão de chance (odds ratio) para não notificação por pediatras de caso de abuso infantil.

\begin{tabular}{|c|c|c|c|c|c|c|c|c|}
\hline \multirow{3}{*}{ Variável } & \multirow{2}{*}{\multicolumn{2}{|c|}{$\begin{array}{c}\text { Identificação } \\
\mathrm{N}=80\end{array}$}} & \multicolumn{2}{|c|}{ Notificação } & \multirow{3}{*}{ OR } & \multirow{3}{*}{$\mathrm{IC} 95 \%$} & \multirow{3}{*}{$\begin{array}{c}\text { OR * } \\
\text { ajustado }\end{array}$} & \multirow{3}{*}{ IC95\% } \\
\hline & & & & & & & & \\
\hline & $\mathrm{N}$ & $(\%)$ & Sim & Não & & & & \\
\hline \multicolumn{9}{|l|}{ Sexo } \\
\hline Masculino & 33 & 41,2 & 26 & 7 & - & - & & \\
\hline Feminino & 47 & 58,8 & 37 & 10 & 1,01 & $0,34-2,98$ & & \\
\hline \multicolumn{9}{|l|}{ Setor de Atividade } \\
\hline Privado e público /público & 69 & 89,61 & 58 & 11 & - & - & - & - \\
\hline Privado (exclusivo) & 11 & 73,33 & 5 & 6 & 6,33 & $1,64-24,42$ & 4,02 & $0,92-17,46$ \\
\hline \multicolumn{9}{|l|}{ Filhos } \\
\hline Não & 28 & 68,2 & 23 & 5 & - & - & & \\
\hline Sim & 52 & 31,8 & 40 & 12 & 1,38 & $0,43-4,41$ & & \\
\hline \multicolumn{9}{|l|}{ Pós-graduação } \\
\hline Mestrado/doutorado & 12 & 15,4 & 10 & 2 & - & - & & \\
\hline Residência & 57 & 71,0 & 48 & 9 & 0,94 & $0,22-5,99$ & & \\
\hline Especialização/nenhuma & 9 & 11,5 & 4 & 5 & 6,25 & $0,44-31,62$ & & \\
\hline \multicolumn{9}{|l|}{ Universidade de graduação } \\
\hline Pública & 65 & 82,2 & 51 & 14 & - & - & & \\
\hline Particular & 14 & 17,8 & 11 & 3 & 0,99 & $0,25-4,11$ & & \\
\hline \multicolumn{9}{|l|}{ Tempo de formado } \\
\hline$<11$ anos & 24 & 31,1 & 21 & 3 & - & - & & \\
\hline $11-20$ anos & 24 & 31,1 & 20 & 4 & 1,4 & $0,28-7,05$ & & \\
\hline$>20$ anos & 29 & 37,8 & 20 & 9 & 3,15 & $0,74-13,33$ & & \\
\hline \multicolumn{9}{|l|}{ Conhecimento } \\
\hline Suficiente & 49 & 61,2 & 43 & 6 & - & - & - & - \\
\hline Insuficiente & 31 & 38,8 & 20 & 11 & 3,94 & $2,06-22,08$ & 5,06 & $1,45-17,59$ \\
\hline \multicolumn{9}{|l|}{ Confiança } \\
\hline Não & 44 & 57,9 & 38 & 6 & - & - & & \\
\hline Sim & 32 & 42,1 & 24 & 8 & 2,11 & $0,65-6,84$ & & \\
\hline \multicolumn{9}{|l|}{ Maus-tratos na infância } \\
\hline Não & 69 & 87,4 & 57 & 12 & - & - & - & - \\
\hline Sim & 10 & 12,6 & 5 & 6 & 4,75 & $1,19-19,02$ & 0,98 & $0,86-1,12$ \\
\hline \multicolumn{9}{|l|}{ Medo de envolvimento legal } \\
\hline $\operatorname{sim}$ & 71 & 96,3 & 62 & 15 & - & - & & \\
\hline Não & 3 & 3,7 & 1 & 2 & 8,27 & $0,71-97,33$ & & \\
\hline
\end{tabular}

OR = Odds ratio 


\section{Discussão}

O estudo aponta primeiramente para um achado contraditório: apesar da alta taxa de desconfiança nos órgãos de proteção e do medo do pediatra de ser envolvido em um processo legal, a identificação e a notificação de casos de maus-tratos infantis foi uma prática freqüente entre os profissionais. A atitude de notificar dependeu significantemente do grau de conhecimento sobre maus-tratos infantis.

Inicialmente, devem ser ressaltadas as limitações deste estudo. Primeiro, não foi possível precisar o número total de casos de maus-tratos identificados e notificados pelos pediatras, nem o setor de atuação do pediatra em que o caso foi identificado. Muitos deles desenvolvem atividade profissional há muitos anos, com trocas de inserção profissional entre privado e público. Nesses casos, para evitar viés de lembrança, questionou-se apenas se haviam notificado algum dos casos identificados e o setor em que estavam atuando no momento. Segundo, não foi possível identificar a magnitude da gravidade. O tipo e a intensidade da gravidade são fatores importantes para a notificação do caso. 4,10 No entanto, não havia diferença significativa nos tipos (dados não apresentados) de maus-tratos identificados entre os pediatras que notificaram e àqueles que não notificaram, supondo-se, portanto, que a incidência de casos graves foi semelhante nos dois grupos. Fora isso, questões culturais, tais como crença religiosa e outras, as quais poderiam estar influenciando atitudes profissionais, não foram avaliadas. Apesar dessas limitações, este estudo foi capaz de identificar os fatores que envolveram o pediatra diante de um caso de violência contra criança, influenciando a sua atitude no momento da notificação.

Alguns fatores estão envolvidos na menor probabilidade de notificação de um caso quando os pediatras atuam exclusivamente no setor privado, tais como: envolvimento emocional e aspectos financeiros (classe social do paciente).5,14 O medo de envolvimento legal, reportado pela grande maioria dos pediatras, poderia refletir-se na ausência de suporte institucional eficaz, inexistente no setor privado. $\mathrm{O}$ isolamento do pediatra privado estaria inibindo atitudes pró-ativas em relação à notificação de casos de maus-tratos infantis. Portanto, os resultados sugerem que o incremento do conhecimento sobre o tema, poderia atuar como facilitador para a notificação de casos suspeitos, amenizando essas dificuldades.

Vários estudos apontam a influência positiva do conhecimento, do acesso a treinamento e da qualificação da formação médica sobre a atitude dos profissionais de saúde em notificar casos de maus-tratos. Os achados desse estudo confirmam essa associação. 3,11,14,15 O grau de conhecimento independe do local e do tipo de inserção do profissional de saúde. Neste sentido, a maioria significativa dos pediatras de Porto Alegre estava habilitada a lidar adequadamente com as situações expostas no instrumento de pesquisa. Contudo, isso não reflete necessariamente o cotidiano do pediatra, pois situações reais poderiam ser conduzidas de maneira distinta às propostas no questionário.

O baixo grau de confiança nos órgãos de proteção têm sido fator relacionado com a não notificação, mesmo em países de notificação mandatória e com sistema de proteção adequado.5,6,914,16 No presente estudo a notificação não foi influenciada pelo grau de confiança nos órgãos de proteção. Esse achado estaria evidenciando uma mudança de atitude do pediatra? Alterações contextuais nas quais o combate à violência infantil tornou-se uma política forte no âmbito da saúde coletiva, com inúmeras estratégias de prevenção e vinculação na mídia, influenciariam positivamente a decisão de notificar. Todavia, a possibilidade da notificação levar a um prognóstico ainda menos favorável faz parte desse cenário.

Outras questões continuam abertas, salientandose a taxa de pediatras que reportam terem sido vítimas de maus-tratos na infância e sua associação com a atitude de não notificar. Esse achado aponta para a direção oposta dos poucos achados que investigaram tal relação. ${ }^{17} \mathrm{O}$ receio de mobilizar sentimentos indesejáveis ligados a uma experiência traumática poderia influenciar a decisão de não notificar um caso de maus-tratos infantis. Porém essa hipótese deve ser explorada em futuras investigações.

Finalmente, prevenir casos de violência infantil ou reduzir suas seqüelas é uma das muitas tarefas do pediatra. Nesse sentido, o incremento de programas de formação continuada, o aprimoramento das instituições de proteção à criança e ao adolescente e a ampliação das redes de suporte profissional poderão reduzir o grau de insegurança profissional e incrementar o número de notificações de casos de maus-tratos. 


\section{Referências}

1. Ministério da Saúde. Notificação de maus-tratos contra crianças e adolescentes pelos profissionais de saúde: um passo a mais na cidadania em saúde. Brasília (DF); 2002.

2. Brasil. Estatuto da criança e do adolescente: Lei 8.069 de 13 de julho de 1990. Brasília (DF): Ministério da Justiça; 1990.

3. Vulliamy AP, Sullivan R. Reporting child abuse: pediatrician's experiences with child protection system. Child Abuse Negl 2000; 24: 1461-70.

4. Warner JE, Hansen DJ. The identification and reporting of physical abuse by physicians: a review and implications for research. Child Abuse Negl 1994; 18: 11-25.

5. Ron S. Pediatrician in Israel: factors which affect the diagnosis and reporting of maltreated children. Child Abuse Negl 1998; 22: 143-53.

6. Assis SG. Crianças e adolescentes violentados: passado, presente e perspectivas para o futuro. Cad Saúde Pública 1994; 10: 126-34.

7. Zellman GL. The impact of case characteristics on child abuse reporting decisions. Child Abuse Negl 1992; 16: 57 74.

8. Gonçalves HS, Ferreira AL. A notificação da violência intrafamiliar contra crianças e adolescentes por profissionais de saúde. Cad Saúde Pública 2002; 18: 315-9.

9. Pollac J, Sheldon L. Countertransference and faillure to report child abuse and neglect. Child Abuse Negl 1989; 13:
$515-22$.

10. Zellman GL. Report decision - making patterns among mandated child abuse reporters. Child Abuse Negl 1990; 14: 325-36.

11. Finkelhor D, Zellman GL. Flexible reporting options for skilled child abuse professionals. Child Abuse Negl 2000; 15: 335-41.

12. Almeida EC. Violência doméstica: um desafio para a formação do pediatra [dissertação mestrado]. Rio de Janeiro: Universidade do Estado do Rio de Janeiro; 1998.

13. Oppenheim AN. Questionaire design, interviewing and attitude measurement. London: Pinter; 1992.

14. Haeringen ARV, Dadds M, Armstrong KL. The child abuse lottery - will the doctor suspect and report? Physician attitudes towards reporting of suspected child abuse and neglect. Child Abuse Negl 1998; 22: 159-69.

15. Escobar SF. A study of factors influencing child abuse reporting by mental health-professionals [tese doutorado]. Illinois: University of Illinois; 1995.

16. Braz M, Cardoso MHCA. Em contato com a violência - os profissionais de saúde e seus pacientes vítimas de maustratos. Rev Latinoamer Enf 2000; 8: 91-97.

17. Socolar RRS, Reives P. Factors that facilitad or imped physicians who perform evoluations for child maltratement. Child Malt 2002; 7: 377-81.

Recebido em 23 de agosto de 2004

Versão final apresentada em 14 de dezembro de 2004

Aprovado em 5 de janeiro de 2005 